\title{
Identification and Characterization of Skin Biomolecules for Drug Targeting and Monitoring by Vibrational Spectroscopy
}

\author{
Natalja Skrebova Eikje ${ }^{*}, 1$ Katsuo Aizawa ${ }^{1}$, Takayuki Sota ${ }^{1}$, Yukihiro Ozaki $^{2}$ and Seiji Arase ${ }^{3}$ \\ ${ }^{I}$ Department of Electrical Engineering and Bioscience, Waseda University, Tokyo, Japan \\ ${ }^{2}$ Department of Chemistry, School of Science and Technology, Kwansei-Gakuin University, Hyogo, Japan \\ ${ }^{3}$ Department of Dermatology, School of Medicine, Tokushima University, Tokushima, Japan
}

\begin{abstract}
The article discusses the application of vibrational spectroscopy techniques for in vivo identification and characterization of glucose biomolecules monitored in the skin of healthy, prediabetes and diabetes subjects; for molecular characterization of water and proteins in in vivo monitored patch tested inflamed skin of the patients with contact dermatitis; for description of nucleic acids and proteins at the molecular level with progression to malignancy in skin cancerous lesions. The results of the studies show new possibilities to assess activity levels of glucose metabolism in the skin tissue of healthy, prediabetes and diabetes subjects; activity and severity of inflammation; activity of the processes of carcinogenesis with regard to benign, premalignant and malignant transformation. Based on our findings, we suggest that vibrational spectroscopy might be a rapid screening tool with sufficient sensitivity and specificity to identify and characterize skin biomolecules in described diseases for drug targeting and monitoring by the pharmacological community.
\end{abstract}

Keywords: Vibrational spectroscopy, Raman spectroscopy, infrared spectroscopy, skin biomolecules, molecular diagnosis, molecular characterization, skin monitoring, skin cancer, skin inflammation, skin glucose.

\section{INTRODUCTION}

Vibrational spectroscopy, both infrared (IR) and Raman, is a well-known structural and analytical laboratory method, because of the high sensitivity of vibrational spectra to characterize structures of biological molecules - water, proteins, nucleic acids, lipids, carbohydrates, etc. [1]. Due to the fact that IR and Raman spectroscopies are also highly sensitive to detect structural changes with the diseased state, vibrational spectroscopy has recently received much attention from the medical community, including dermatology, as a new promising tool for rapid, highly-informative, non-destructive, qualitative and (semi-) quantitative molecular characterization of normal and pathological tissues in vitro, ex vivo and in vivo [1-7].

The skin is a very complex and heterogeneous tissue. However, as the human body, on a simple level, is composed primarily of biomolecules of water, proteins, nucleic acids, lipids and carbohydrates, the relative ratios and the absolute concentrations of each of them can be determined in vibrational spectra.

Isolated biomolecules of the nucleic acids, water, proteins, lipids, carbohydrates and others have been intensively studied and well-described by vibrational spectroscopy techniques [1].

IR spectroscopy has an advantage to identify and characterize nucleic acids, in comparison to Raman

*Address correspondence to this author at the Department of Electrical Engineering and Bioscience, Waseda University, 3-4-1 Ohkubo, Shinjukuku, Tokyo 169-8555, Japan;

E-mail: eikjen@yahoo.co.uk; peikje@hotmail.com spectroscopy, which provides information related to individual nucleotides only. So, IR spectra in the $1620-1750 \mathrm{~cm}^{-1}$ region corres-ponds to in-plane double-bond vibrations of the bases; the $1230 \mathrm{~cm}^{-1}$ and $1090 \mathrm{~cm}^{-1}$ bands are assigned to antisymmetric and symmetric phosphate stretching vibrations, respectively; ribose has a strong C-O band at $1120 \mathrm{~cm}^{-1}[1,3]$.

Both IR and Raman provide information on the secondary structure of proteins, ligand interactions and folding. In the vibrational spectra, amide I in the $1600-1700 \mathrm{~cm}^{-1}$ region is by far the best characterized, which is due mostly to the $\mathrm{C}=\mathrm{O}$ stretching vibrations of the peptide backbone. The amide II band in the $1480-1575 \mathrm{~cm}^{-1}$ region, due largely to a coupling of $\mathrm{CN}$ stretching and in-plane bending of $\mathrm{N}-\mathrm{H}$ group, is strong in IR, but extremely weak in Raman. The amide III band in the $1230-1300 \mathrm{~cm}^{-1}$ region, arising from coupling of C-N stretching and N-H bending, is fairly weak in IR but quite strong in Raman $[1,3]$.

The major absorption bands of lipids are at 1738, 1465, $1255,1168,1095,1057$ and $968 \mathrm{~cm}^{-1}[1,3]$.

Sugars are the most common carbohydrates. They are presented in biological media primarily as hexose sugars, such as glucose, and as pentose sugars, mainly as the ribose component of the nucleic acid backbone of DNA and RNA. Polysaccharides in the body are found either in a free state or combined with proteins, in a complex known as glycoproteins. The only polysaccharide in the body that is not bound to a protein is glycogen, the spectrum of which is highly dependent on the degree of hydration. The major absorption bands of glycogen in solution are observed in IR spectra at 1153, 1105, 1082, 1043, 1025, 996 (weak shoulder) and 931 $\mathrm{cm}^{-1}$. The corresponding bands for the solid spectrum are 
1149, 1078, 1043 (weak shoulder), 1016, 996 and $931 \mathrm{~cm}^{1}$ [1].

As a consequence of the complexity of skin tissue, comprising several components and various cell types, the band assignment of different biomolecules in vibrational spectra of the skin is difficult, especially in the diseased state, because it is influenced by numerous intermolecular interactions. Therefore, it is important not only to identify (facilitate) and compare the band assignment of spectra of major constituents of skin and its compounds in normal and pathological tissue, but also to molecularly characterize them with regard to numerous skin diseases and conditions. Since all diseased states and conditions, without exception, are caused by fundamental alterations in cellular and/or tissue biochemistry due to specific changes in one or all biomolecular components, then these changes should be reflected in vibrational spectra and, in principle, may to be further used as phenotypic markers of the diseases for diagnoses, drug targeting and monitoring $[1,6]$.

Such an approach would be much needed in the new fields of applications, such as the individualization of drug or treatment in certain diseases and monitoring the effects of therapies, based on the already demonstrated potential of IR and Raman spectroscopies to non-invasively diagnose and continuously monitor patients in the clinical environment. At a more fundamental level, the in-depth analysis of the wealth of information contained in IR- and Raman spectra of certain skin diseases about specific molecular composition and structure can be used not only to further explore pathological processes and their progression, but also to further transform for directing real-time therapeutic intervention.

In this article we discuss the state of the art of applying vibrational spectroscopy techniques for in vivo identification and characterization of glucose biomolecules monitored in the skin of healthy, prediabetes and diabetes subjects; for molecular characterization of water and protein biomolecules in in vivo monitored patch tested inflamed skin of the patients with contact dermatitis; for description of nucleic acids and proteins at the molecular level with progression to malignancy in skin cancerous lesions. The intended reader is directed to recent review and research articles that address these issues in more detail [3, 8-11]. Rather, we would like to take the perspective of the intended end user of described vibrational spectroscopy techniques from pharmaceutical community. The path toward implementing vibrational spectroscopy technique for molecular drug targeting and monitoring is discussed, based on how far have we progressed in our studies.

\section{SUBJECTS AND METHODOLOGY}

Very few vibrational spectroscopy techniques have been translated into patient's health care in dermatological clinics, in spite of the rapid developments and progress in technology for using them in laboratories [10]. Mainly, it is because measuring of the skin requires certain technical prerequisites, like availability of remote fiber optic probes able to measure at any anatomical location, availability of the desired measuring aperture area in the used instrumentation, short measuring time, appropriate wavelength for laser light excitation in order to overcome skin tissue autofluorescence, and measurement of skin spectra with a high (adequate) signal-to-noise ratio (SNR). Moreover, it is important to use an appropriate method with desired depth penetration of skin tissue for the study, so that it is relevant with regard to the skin layer origin of investigated disease or a (patho-)physiological condition in question $[3,8,10]$. Based on these issues, the FT-IR spectroscopy with the ATR (attenuated total reflection) accessory was chosen for our study to in vivo identify and characterize glucose biomolecules in monitored skin of healthy, prediabetes and diabetes subjects; NIR FT Raman spectroscopy technique with a remote fiber optic probe to molecularly characterize and in vivo monitor skin inflammation on the back of patch tested patients with contact dermatitis; and FT-IR spectroscopy with a microscope accessory to in vitro characterize spectra of benign, premalignant and malignant skin lesions at the molecular level, which is discussed below in detail.

\section{ATR-FTIR Spectroscopy for Real-Time Non-Invasive Skin Glucose Characterization and Monitoring}

A high throughput ATR accessory, the PIKE Technologies Horizontal ATR (HATR) (ATR-8200 HA), is designed for use in commercially available Shimadzu FTIR spectrometer (Shimadzu IRPrestige-21/FTIR-8400S, Japan). The ATR accessory is available with two basic types of mounted crystal, the trough plate and the flat plate, both is of a trapezoid shape and is $80 \mathrm{~mm}$ long, $10 \mathrm{~mm}$ wide and $4 \mathrm{~mm}$ thick. This thickness of the crystal was carefully chosen by manufacturer in order to produce optimum performance from the accessory, and the crystal dimensions have been chosen to maximize SNR in the resulting spectra. To perform in vivo measurements from the inner wrists of the subjects, the flat plate has been used for the collection of mid-infrared spectra in our study. Before each measurement the spectrum was obtained by rationing a scan measured from the inner wrist area to a background scan. In vivo infrared ATR spectra were recorded in the $700-4000 \mathrm{~cm}^{-1}$ wavenumber region at a resolution of $4 \mathrm{~cm}^{-1}$, using number 20 of accumulation for the collection of interferogram.

Eleven subjects participated in the study: one with diabetes (type 2); three, as suspects of having impaired glucose tolerance (prediabetes), due to randomly measured postprandial hyperglycemia; and seven healthy subjects. The following measurements were done: 1) randomly, i.e. casually measured regardless of when the patient last ate; 2) on mornings, when a subject has refrained from eating for 10 to 16 hours (fasting measurements); 3) measured from 1 to 3 hours after a meal (postprandial measurements); 4) during OGTT (oral glucose tolerance test), after given orally a glucose solution to drink, including $5 \mathrm{~g}$ (minimum dose), $20 \mathrm{~g}$ (medium dose) and/or $75 \mathrm{~g}$ (maximum dose, i.e. clinical test dose) of glucose.

All measurements by ATR-FTIR spectroscopy were performed immediately after determining blood glucose level by a portable glucosemeter (SKK GluTestS, Sanwa Chemical Institute, Nagoya, Japan) in mg/dL. 
The techniques applied to treat in vivo HATR-FTIR spectra included: 1) normalization to amide I; 2) multiple baseline correction; 3 ) in the present study, the amide I band near $1645 \mathrm{~cm}^{-1}$ was considered constant, and therefore was used in calculating the intensities of the peaks at around $1030 \mathrm{~cm}^{-1}$, $1041 \mathrm{~cm}^{-1}, 1080 \mathrm{~cm}^{-1}, 1118 \mathrm{~cm}^{-1}$ and $1153 \mathrm{~cm}^{-1}$.

Fiberoptic NIR FT Raman Spectroscopy for Direct Molecular Characterization and In Vivo Monitoring of Inflammation in the Skin (Type IV Allergic Reaction)

Fiber optic NIR FT RFS 100-Raman spectrometer on a Bruker IFS 66 optics system (Bruker Analytik GmbH, Karlsruhe, Germany), equipped with a liquid nitrogen-cooled Ge detector, was using the excitation source of a $1064 \mathrm{~nm}$ line (virtually elicits almost no fluorescence) from a continuous wave Nd:YAG laser at $200 \mathrm{~mW}$. In vivo Raman spectra of normal control and skin reacting to patches (inflamed skin) on the back of the patients, as suspects of contact dermatitis, were measured in $400-3500 \mathrm{~cm}^{-1}$ region within 250 scans by using a resolution of $8 \mathrm{~cm}^{-1}$.

In the present study fiberoptic NIR FT Raman spectroscopy was used to instrumentally assess 63 positive patch test (PT) reactions at $48 \mathrm{~h}$ and $72 \mathrm{~h}$, at the time of their readings in a contact dermatitis clinic. The application of commercially available allergens for $48 \mathrm{~h}$ was used to clinically reproduce contact dermatitis, in miniature, in the patients suspected to have this disease. The common method of clinical reading includes scoring into irritant, doubtful (+?; faint erythema only), weak (+; erythema, oedema, possibly papules), strong (++; erythema, oedema, papules, vesicles) and extremely strong $(+++$; intense erythema and oedema; coalescing vesicles) positive PT reactions [12].

For assessment of water content in the skin, the intensity of an $\mathrm{O}-\mathrm{H}$ stretching mode of water at $3250 \mathrm{~cm}^{-1}$, that is presented in vivo Raman spectra in a broad feature between 3100 and $3500 \mathrm{~cm}^{-1}$, was used in the calculated ratio $I_{3250} / I_{2940}[13,14]$.

The Wilcoxon Rank-Sum test, a nonparametric MannWhitney U-test was used to compare different PT reaction groups at $48 \mathrm{~h}$ and $72 \mathrm{~h}$. A value of $p<0.05$ was considered to indicate a statistically significant difference.

\section{FT-IR Microspectroscopy for In Vitro Description of Skin Cancerous Tissue}

An FT-IR microspectrometer (IR-MAU200, JEOL Co., Tokyo, Japan) was used to obtain spectra with a resolution of $4 \mathrm{~cm}^{-1}$ over a spectral range of $800-4000 \mathrm{~cm}^{-1}$, using a knife edge aperture reduced to a $25 \times 25 \mu \mathrm{m}$. Spectra of 47 normal and lesional, epidermal (Bowen's disease, solar keratosis, basal cell carcinoma (BCC), squamous cell carcinoma (SCC), malignant melanoma (MM)) and dermal (intradermal nevus), skin components were acquired from a $6 \mu \mathrm{m}$ cutsections on $\mathrm{a} \mathrm{CaF}_{2}$ (calcium fluoride) slide glasses, with speed scans of 127 [15].

\section{RESULTS}

ATR-FTIR Spectroscopy for In Vivo Identification, Characterization and Monitoring of Glucose Biomolecules in the Skin of Healthy, Prediabetes and Diabetes Subjects

In all measured volunteers a closer assessment of the glucose-related region between 1000 and $1180 \mathrm{~cm}^{-1}$ in ATRFTIR spectra found glucose-specific peaks at about 1030 $( \pm 2-3) \mathrm{cm}^{-1}, 1041( \pm 4) \mathrm{cm}^{-1}, 1080( \pm 4) \mathrm{cm}^{-1}, 1118(-7$; + 4) $\mathrm{cm}^{-1}$ and $1153( \pm 4) \mathrm{cm}^{-1}$.

In vivo spectra of monitored skin in 8 subjects during OGTT showed shifts, at most at $1041 \mathrm{~cm}^{-1}$ and at $1118 \mathrm{~cm}^{-1}$ peaks, and at less at $1030 \mathrm{~cm}^{-1}, 1080 \mathrm{~cm}^{-1}$ and at $1153 \mathrm{~cm}^{-1}$ peaks. Shifts to the left were more seen in healthy volunteers and shifts to the right were noticed in prediabetes and diabetes subjects (see peak captions in Fig. 2a-c).

Fig. (1) demonstrates examples of spectral variations of the intensity of the peaks at $1041 \mathrm{~cm}^{-1}$ and at $1118 \mathrm{~cm}^{-1}$ in the skin of the patients with type 2 diabetes, prediabetes and a healthy measured subject. In vivo spectra of all measured prediabetes and diabetes volunteers showed the prominence of the peak near $1118 \mathrm{~cm}^{-1}$ over the peak intensity near 1041 $\mathrm{cm}^{-1}$, that was not seen in healthy subjects. Therefore, based on the differences of the intensities near peaks at $1041 \mathrm{~cm}^{-1}$ and at $1118 \mathrm{~cm}^{-1}$, two spectral patterns could be seen: $I_{1118}>$ $I_{1041}$ for prediabetes and diabetes subjects, and $I_{1118}<I_{1041}$ for non-diabetes subjects. These differences were seen independently on the screening tests performed.

Real-time non-invasive monitoring of absorbances of the glucose peak near $1030 \mathrm{~cm}^{-1}$ during a 4-hour OGTT after intake of a clinical dose of glucose $(75 \mathrm{~g})$ in the patient with type 2 diabetes demonstrates an ability to monitor glucose activity changes in the skin tissue by ATR-FTIR spectroscopy in vivo (Fig. 2).

Fig. (3a-c) demonstrates examples of in vivo skin glucose monitoring based on calculated absorbances of glucose biomolecules at wavelengths of $1041 \mathrm{~cm}^{-1}, 1080 \mathrm{~cm}^{-1}, 1118 \mathrm{~cm}^{-1}$ and $1153 \mathrm{~cm}^{-1}$ during a 1 -hour OGTT $(75 \mathrm{~g})$ at a $15-\mathrm{min}$ interval in a healthy, prediabetes and diabetes subjects. It also shows evidences of indirect correlation between the intensities of these peaks and blood glucose levels in a timedependent manner (Fig. 3d). During OGTT this in vivo monitoring revealed that specific for a healthy, prediabetes and diabetes subjects levels of each glucose molecule activity changed at different time-points by means of measured intensities of the peaks at $1041 \mathrm{~cm}^{-1}, 1080 \mathrm{~cm}^{-1}, 1118 \mathrm{~cm}^{-1}$ and $1153 \mathrm{~cm}^{-1}$. Moreover, based on the intensities of the peaks at $1041 \mathrm{~cm}^{-1}$ and $1118 \mathrm{~cm}^{-1}$ we could again recognise 2 spectral patterns in monitored spectra of healthy and diabetes subjects.

Fig. (4) shows clear differences between mean values of the peak near $1030 \mathrm{~cm}^{-1}$ in a dose-dependent manner, measured in the patient with type 2 diabetes during a 30 -minute OGTT monitoring after taking $5 \mathrm{~g}, 20 \mathrm{~g}$ and $75 \mathrm{~g}$ of glucose orally. 


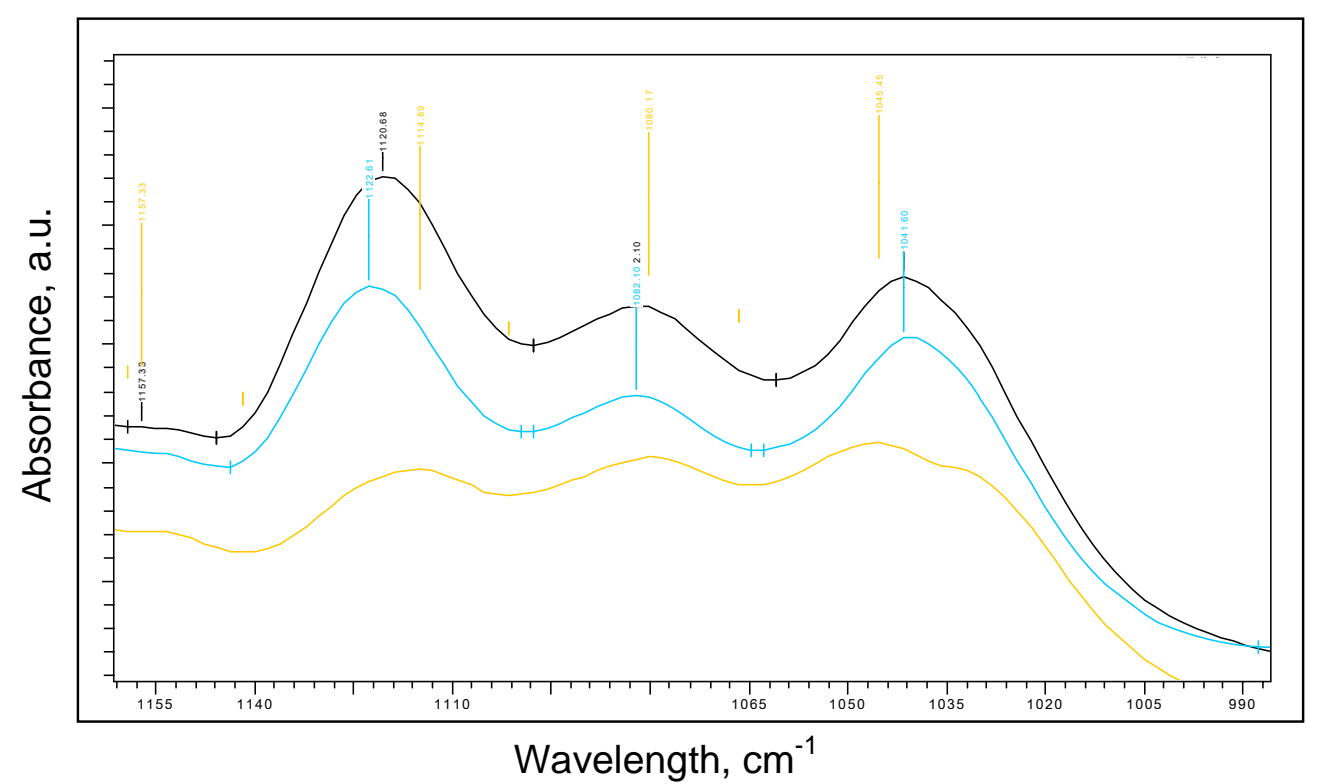

Fig. (1). Two spectral patterns: pattern 1 for prediabetes (blue line)/diabetes (black line) $\left(I_{1118}>I_{1041}\right)$ and pattern 2 for a healthy subject (yellow line) $\left(I_{1118}<I_{1041}\right)$.

\section{Fiberoptic NIR FT Raman Spectroscopy for Molecular Diagnosis and In Vivo Monitoring of Inflammation in the Skin of Patch Tested Patients with Contact Dermatitis}

The most significant changes in the spectra were detected in the regions specific for water content $\left(2800-3400 \mathrm{~cm}^{-1}\right)$ and protein structure $\left(600-1800 \mathrm{~cm}^{-1}\right)$ in all positive PT reactions at $48 \mathrm{~h}$ and $72 \mathrm{~h}$, when compared to normal skin.

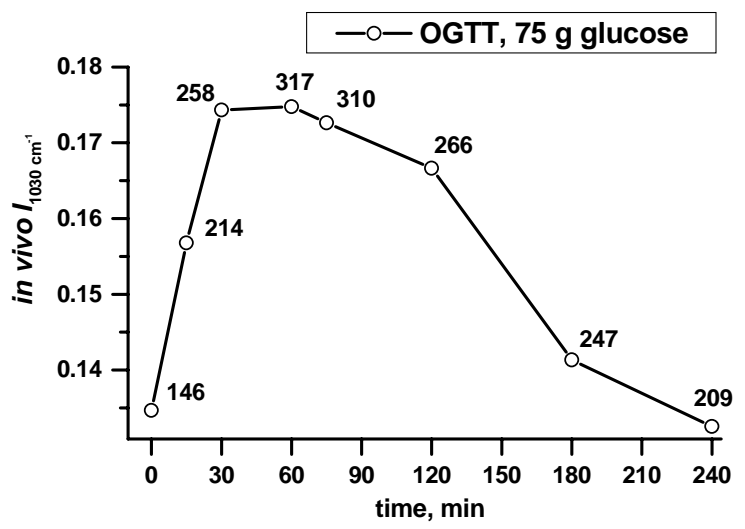

Fig. (2). Example of non-invasive glucose monitoring in the patient with type 2 diabetes by means of calculated intensities of the peak near $1030 \mathrm{~cm}^{-1}$, that are indirectly followed by blood glucose level changes (shown in numbers along the line at determined time intervals) [11, copyright permission for reproduction is obtained $]$.

Based on these spectral changes, primarily spectra of all positive PT reactions were divided into 2 groups: mean spec- tra of irritant (IR), doubtful $(+$ ?) and weak $(+)$ positive PT reactions and mean spectra of strong $(++)$ and extremely strong $(+++)$ positive PT reactions (Fig. 5a-b and Fig. 6a-b). By means of measured differences in absolute values of the $3250 \mathrm{~cm}^{-1}$ peak (ratio $I_{3250} / I_{2940}$ ) mean Raman spectra of [IR; + ?; +] positive PT reactions were calculated as 0.37 (normal control $)<0.42(48 \mathrm{~h})<0.51(72 \mathrm{~h})$, and mean Raman spectra of $[++;+++]$ were calculated as 0.37 (normal control) < $0.59(48$ h) $<0.58$ (72 h) (Fig. 5a-b). Protein structure changes differed mostly between 2 groups of positive PT reactions after $72 \mathrm{~h}$, showing stable decrease at $48 \mathrm{~h}$ and $72 \mathrm{~h}$ at $1244 \mathrm{~cm}^{-1}$ (amide III) and at $937 \mathrm{~cm}^{-1}$ (protein backbone vibration) for the $[++;+++]$-group and coming back to normal at $72 \mathrm{~h}$ after transient decrease at $48 \mathrm{~h}$ for $[\mathrm{IR} ;++$ ? + $]$ group (Fig. 6a-b).

The measurement of water content with further calculation of the ratio $I_{3250} / I_{2940}$ for mean degrees of all reactions was $0.31(-)<0.34(+?)<0.41(+)<0.59(++/+++)$ at $48 \mathrm{~h}$ and $0.31(-)<0.40(+?)<0.44(+)<0.69(++/+++)$ at $72 \mathrm{~h}$. The results of the Mann-Whitney U-test showed significant differences between $(-)$ and (+?) $[p<0.03 / 48 \mathrm{~h}],(+?)$ and $(+)$ $[p<0.04 / 48 \mathrm{~h} ; p<0.01 / 72 \mathrm{~h}],(+)$ and $(++/+++)[p<0.01 / 48$ $\mathrm{h} ; p<0.01 / 72 \mathrm{~h}$ ], but not between negative and doubtful PT reactions $[p>0.05]$ at $72 \mathrm{~h}[12]$ (Fig. 7a-b).

FT-IR Microspectroscopy for In Vitro Molecular Description of Skin Cancerous Tissue

Representative FT-IR spectra in the $800-1750 \mathrm{~cm}^{-1}$ region of epidermal premalignant (Bowen`s disease, solar kerato- 
sis), malignant (SCC, BCC, MM) tumours and dermal benign (nevi) lesions, in comparison to normal epidermal and dermal skin components showed specific and similar progression towards malignancy spectral features: absorption between 1000 and $1150 \mathrm{~cm}^{-1}$ seemed to correlate with a variation of the amide $\mathrm{I}_{1600-1700} /$ amide II ${ }_{1480-1575}$ intensity ratio; the spectral features due to DNA and amide III (965 $\mathrm{cm}^{-1}, 1071 \mathrm{~cm}^{-1}, 1084 \mathrm{~cm}^{-1}, 1095 \mathrm{~cm}^{-1}, 1245 \mathrm{~cm}^{-1}$ ) have been modified and enhanced with progression to malignancy. These are shown in Fig. (8a-d).

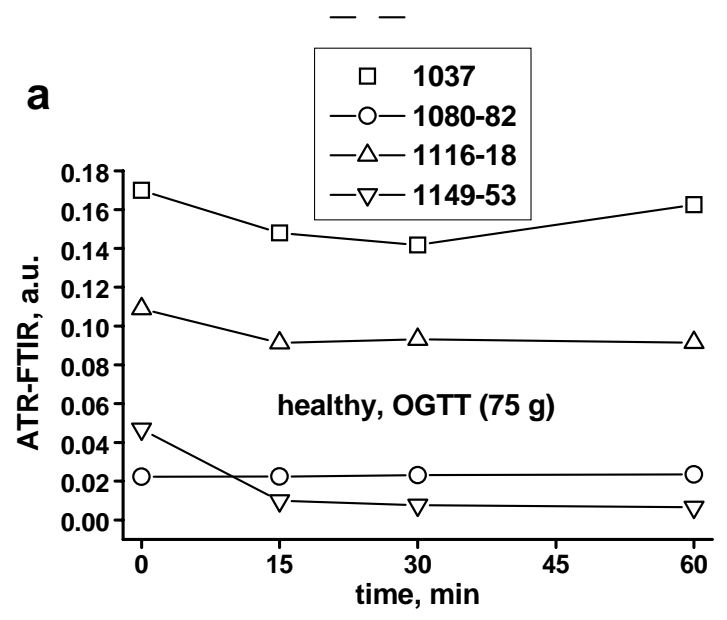

b

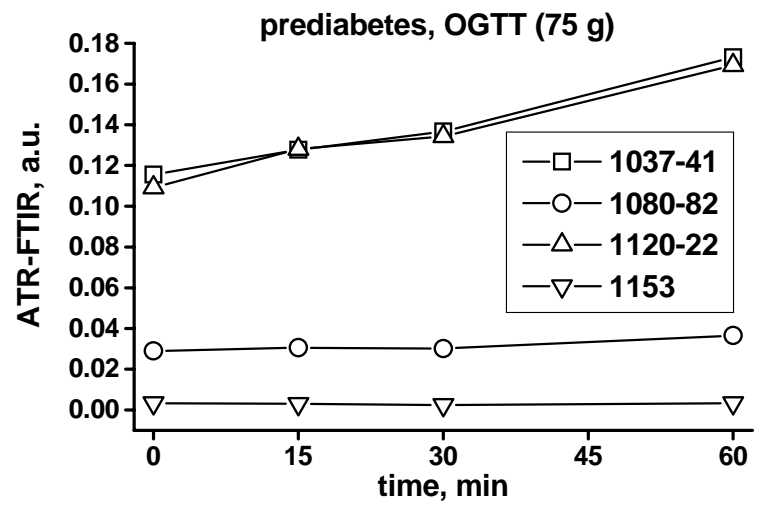

C

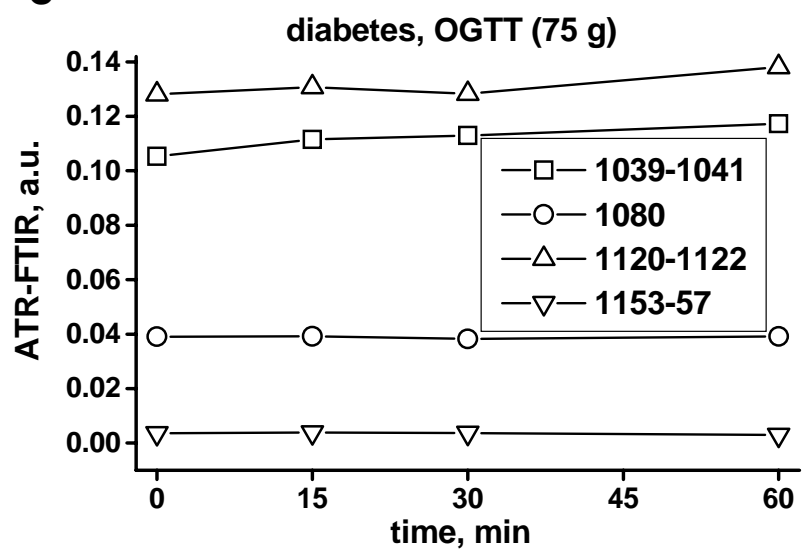

Fig. (3a-c). Examples of non-invasive monitoring of the activities of skin glucose biomolecules at around $1041 \mathrm{~cm}^{-1}, 1080 \mathrm{~cm}^{-1}, 1118$ $\mathrm{cm}^{-1}$ and $1153 \mathrm{~cm}^{-1}$ in the healthy (a), prediabetes (b) and diabetes (c) subjects during a 1-hour OGTT (75 g) at a 15-minute assessments.

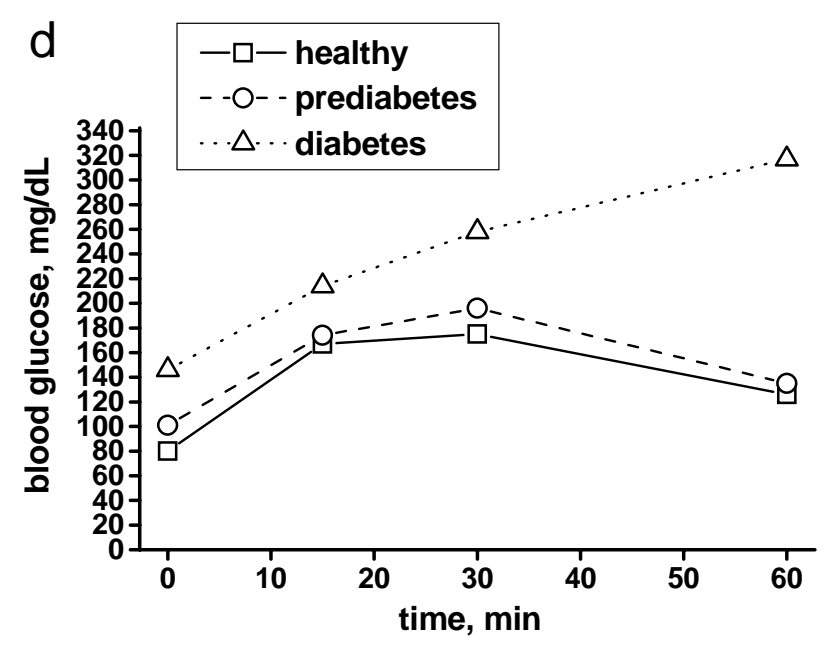

Fig. (3d). Blood glucose levels of healthy, prediabetes and diabetes subjects during a 1-hour OGTT $(75 \mathrm{~g})$ with assessment every 15 minutes.

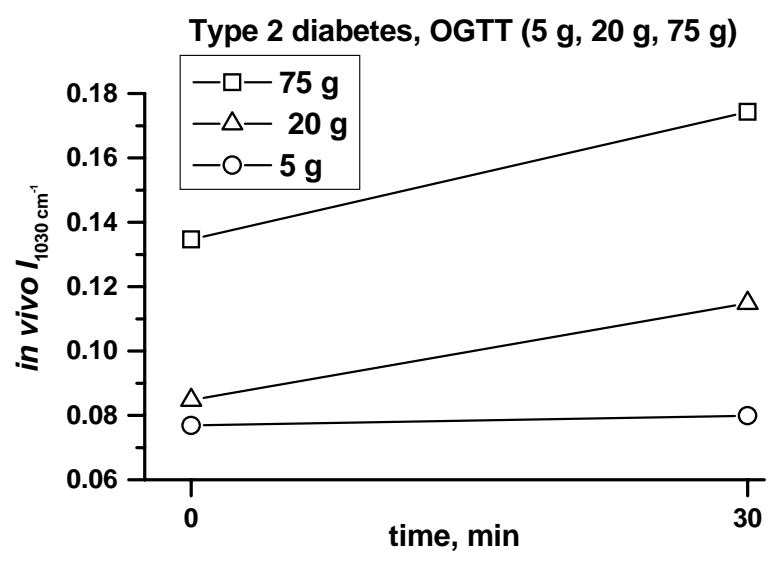

Fig. (4). In vivo monitored increased absorbances of the peak at $1030 \mathrm{~cm}^{-1}$ correlated with different oral doses of glucose $(5 \mathrm{~g}, 20 \mathrm{~g}$, $75 \mathrm{~g}$ ) and blood glucose level changes assessed at $30 \mathrm{~min}$ of OGTTs in the patient with type 2 diabetes [11, copyright permission for reproduction is obtained].

\section{DISCUSSION}

Our studies demonstrate that applied vibrational spectroscopy techniques for identification, characterization and monitoring of skin biomolecules were not only able to noninvasively identify and comparatively characterize qualitatively and quantitatively skin biomolecules in described skin diseases and conditions, but were also able to continuously monitor pathophysiological processes in the skin with regard to the assessment of studied skin diseases and conditions, and their activity and severity at the molecular level.

Although both IR and Raman vibrational spectroscopy techniques are shown to be complementary in identification, characterization and monitoring of skin biomolecules in a rapid, objective and reliable way, the results of our studies certainly demonstrate the relative advantages and disadvantages for measuring described skin diseases and conditions in vitro or in vivo. 

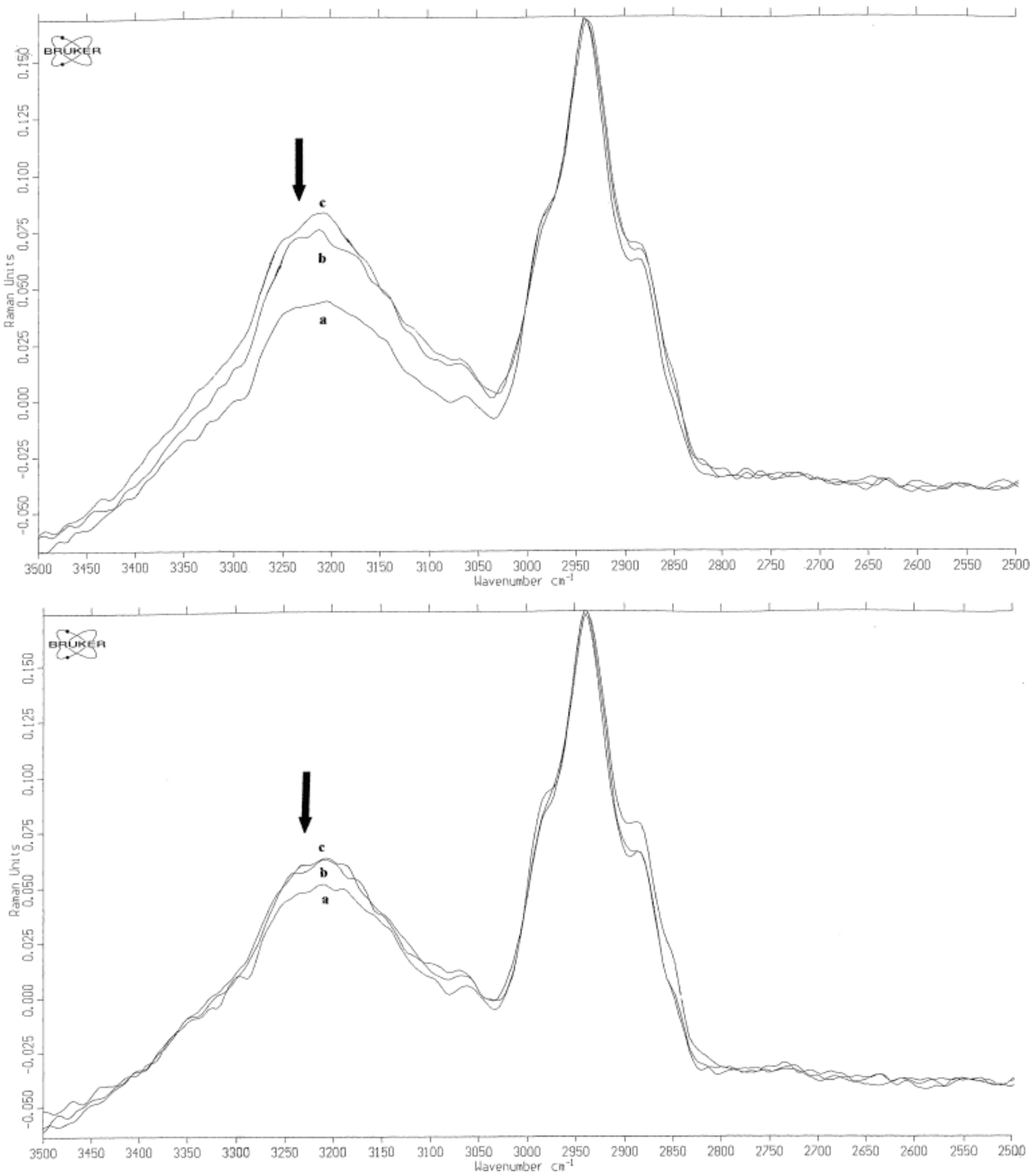

Fig. (5a-b). In vivo NIR FT Raman spectra (mean) in the range of $2500 \mathrm{~cm}^{-1}$ to $3500 \mathrm{~cm}^{-1}$ of normal skin (line a) [++; +++] (Fig. 5a) / [+?; $+? /+;+]$ scored PT reactions (Fig. 5b) at $48 \mathrm{~h}$ (line b) and at $72 \mathrm{~h}$ (line c). The band at $3250 \mathrm{~cm}^{-1}$ is indicated with an arrow [14, copyright permission for reproduction is obtained].

In detail, due to the fact that Raman spectroscopy can be easily applied for the analysis of watery biological samples and due to its demonstrated potential to in vitro characterize structure of water, proteins, lipids and other biomolecules in intact skin [3,7], fiberoptic NIR FT Raman spectroscopy was applied to characterize water and proteins in in vivo monitored inflammed skin directly on the back of the patch tested patients with contact dermatitis. The sensitivity of this method was exploited not only to characterize molecular changes that occurred in skin type IV allergic reactions, but 

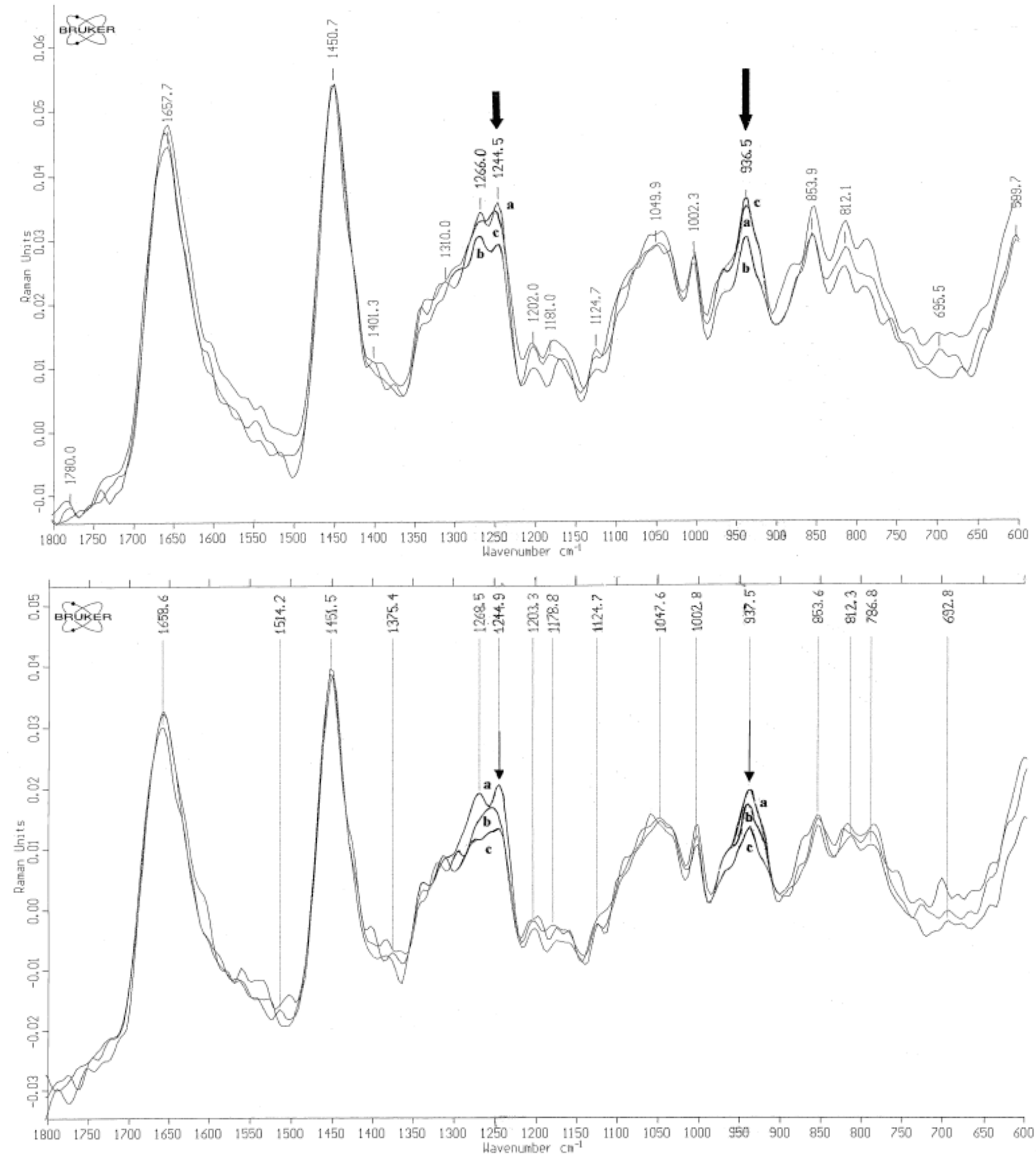

Fig. (6a-b). In vivo NIR-FT Raman spectra over the wavenumber range of 600 to $1800 \mathrm{~cm}^{-1}$ of normal skin (line a) [++; +++] (Fig. 6a) / [+?; $+? /+;+]$ scored PT reactions (Fig. 6b) at $48 \mathrm{~h}$ (line b) and at $72 \mathrm{~h}$ (line c). The bands of collagen amide III at $1244 \mathrm{~cm}^{-1}$ and protein backbone vibration at $937 \mathrm{~cm}^{-1}$ are indicated with arrows [14, copyright permission for reproduction is obtained $]$.

also to monitor these molecular changes as skin progressed from normal to inflamed, as a result of the delayed contact hypersensitivity after application of allergens epicutaneously in patch tests $[8,13,14]$.
Chemical reactions and properties are important in the processes of developing delayed contact hypersensitivity, since it is the interaction of chemicals with self-proteins that drives the delayed allergic response in the skin. In order to cause skin sensitization, a chemical has to penetrate into the 


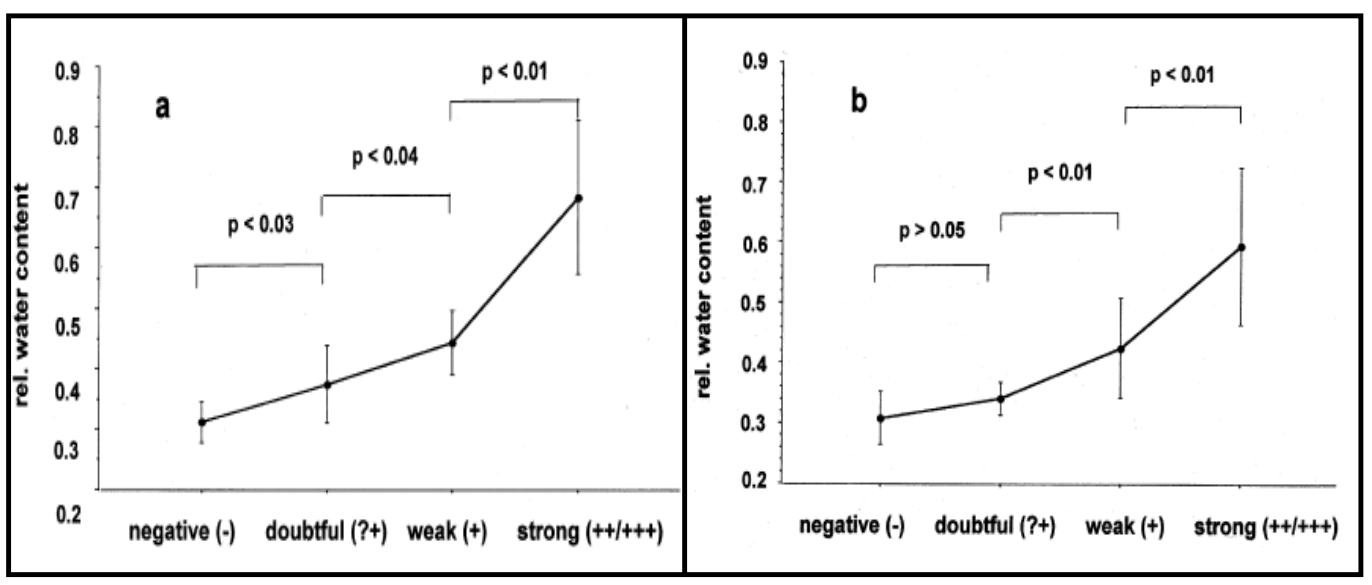

Fig. (7a-b). Mean relative water content values and SD in contrast to visual gradings of PT reactions at $48 \mathrm{~h} \mathrm{(a)} \mathrm{and} \mathrm{at} 72 \mathrm{~h}(\mathbf{b})$.

epidermis and bind to skin components [16, 17]. That was seen in our spectral results on protein structure changes, stable for allergic reactions and transient for irritant, possibly suggesting that amide III and protein backbone vibration could be such allergen-binding proteins [unpublished data].

Moreover, by NIR FT Raman spectroscopy with a remote fiber optic probe the inflammatory oedema of the skin was assessed by means of identification and measurement of absolute values of the $3250 \mathrm{~cm}^{-1}$, specific for water amount and proportional to water content changes in the skin [8]. Based on these calculated differences in various degree of oedema in patch tested skin we were able to continuously monitor the gradual increase of water content in the skin, from the minimal to the most severe, thus providing evidence of suitability of this method for clinical applications [13].

The latter is also confirmed by the fact that in Raman spectra of the skin the majority of Raman scattering light is gathered from the dermis [18], where inflammatory oedema is mainly found. This is also because of the absorption of the long-wave excitation laser (1064 $\mathrm{nm}$ ) and NIR Raman radiation in deeper layers of the skin tissue, thus making perfectly possible in vivo measurements of patch test reactions on the back of the patients, where the thickness of normal skin is approximately $4 \mathrm{~mm}$ [19]. With a remote fiber optic probe attached, that uses the measuring aperture area of $8 \mathrm{~mm}$ in diameter, the patch test size of $1 \mathrm{~cm}^{2}$ was analyzed entirely in our study [8].

All these makes fiberoptic NIR Raman spectroscopy far superior for medical applications not only to clinically investigate cutaneous water distribution and structure by means of identification, characterization and monitoring of water biomolecules in the diseased conditions, but also to in vivo study of essentially unknown molecular mechanisms of contact dermatitis/eczema directly on the patients.

IR light has limited opportunities for tissue penetration, but it has an advantage over Raman spectroscopy to characterize in IR spectra molecules of the nucleic acids and glucose [1]. Due to that, we probed FT-IR microspectroscopy to molecularly characterize skin cancerous tissue in vitro and
ATR-FTIR spectroscopy to characterize skin glucose molecules in the skin in vivo.

Although all biomolecules are important, the nucleic acids DNA and RNA are especially important because they carry within their structure the hereditary information that determines the identity and structure of proteins [1]. They play an important role in carcinogenesis. Understanding the molecular, cellular and tissue changes that occur during skin carcinogenesis is central to cancer research in dermatology. The translational aspects of this field - the development of clinical applications in dermatology from the laboratory findings - aim at improving clinical diagnosis, monitoring and treatment of skin cancer [3]. Clinically differential diagnosis of skin tumours is often difficult and a histopathological diagnosis remains the "gold standard" [1,3]. IR microspectroscopy has already been reported to make a correlation between spectra and histology, with the additional advantage that IR spectra is sensitive toward differentiation, maturation, cell cycle dependence and state of health of cells in tissue [3]. The results of our study on molecular characterization of several benign, premalignant and malignant skin tumours showed not only distinct differences in the shape and position of the nucleic acid absorptions, but also a significant increase in intensities with progression to malignancy. Alterations were also observed in the regions responsible for proteins, which seemed to correlate with the variation of absorption of the peaks in the $1000-1150 \mathrm{~cm}^{-1}$ region, responsible for DNA and amide III [15]. Therefore, it is most probable that malignant transformation triggers similar molecular changes.

With respect to complexity of IR spectra, even within grouped lesion categories, against huge inter- and intrasample variability, FT-IR microspectroscopy has demonstrated an extremely high sensitivity not only to molecularly characterize skin cancerous tissue, but also to detect changes specific for carcinogenesis [15].

ATR-FTIR spectroscopy is an established method to study molecular components and their differences in the uppermost layer of the skin, stratum corneum, in vivo [20]. 

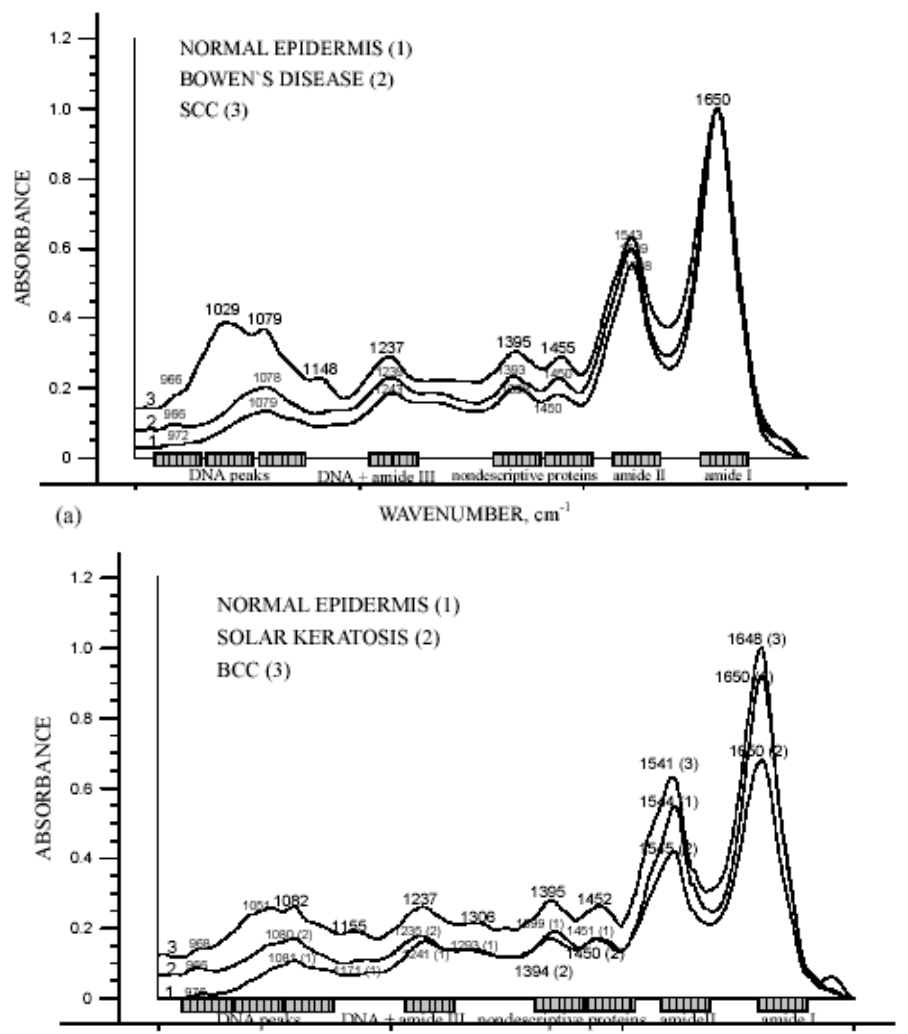

(b)

WAVENUMBER, $\mathrm{cm}^{-1}$

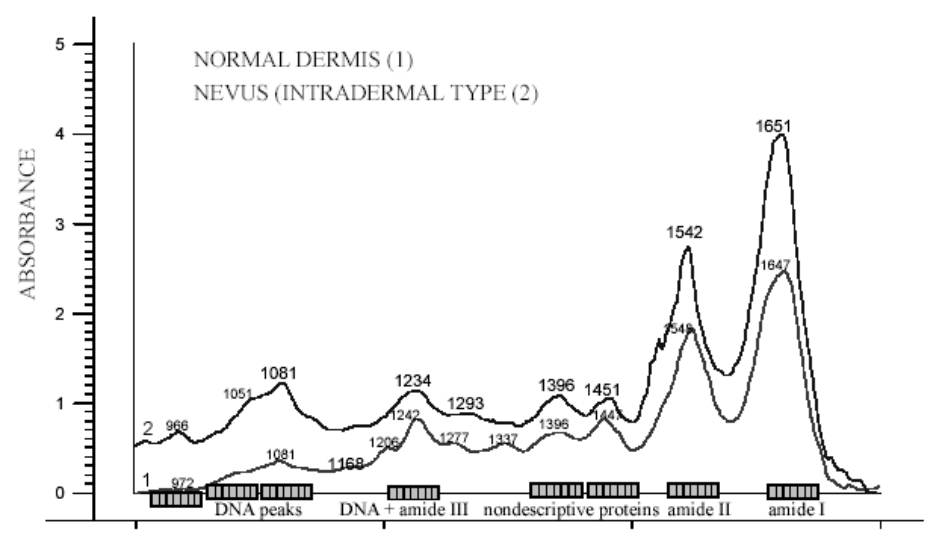

(c)

WAVENUMBER, $\mathrm{cm}^{-1}$

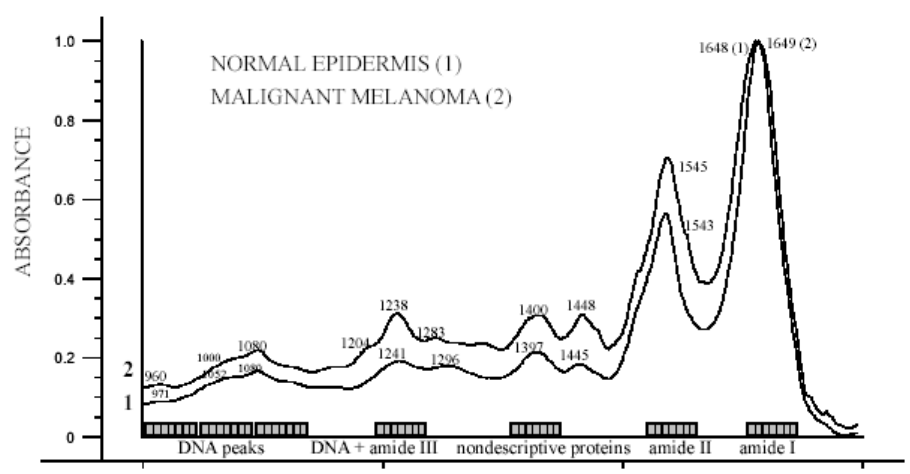

(d)

WAVENUMBER, $\mathrm{cm}^{-1}$

Fig. (8a-d). Representative FT-IR spectra over the wavenumber range from 800 to $1750 \mathrm{~cm}^{-1}$ of epidermal premalignant (Bowen`s disease, solar keratosis), malignant (SCC, BCC, MM) tumours and dermal benign (nevi) lesions, in comparison to normal epidermal and dermal skin components [15, copyright permission for reproduction is obtained]. 
Although early studies intensively describe in vivo regional variations and depth-dependent non-homogeneity in the $1000-4000 \mathrm{~cm}^{-1}$ region, so far the glucose-specific region between $1000 \mathrm{~cm}^{-1}$ and $1180 \mathrm{~cm}^{-1}$ has not been fully described in the literature. [11] In our study HATR-FTIR has been probed to in vivo identify and characterize molecules of glucose in the skin tissue from the inner wrists of measured non-diabetes and (pre-)diabetes subjects.

Spectral results from 7 healthy subjects identified glucosespecific peaks at about $1030 \mathrm{~cm}^{-1}, 1041 \mathrm{~cm}^{-1}, 1080 \mathrm{~cm}^{-1}, 1118$ $\mathrm{cm}^{-1}$ and $1153 \mathrm{~cm}^{-1}$ under different screening test conditions. Although IR spectra of studied absorbances of glucose at a wavelength of $1030 \mathrm{~cm}^{-1}$ is well-known for its good correlation with a range of glucose concentrations in vitro [11], the behaviour of that glucose molecule in the skin in vivo is remained to be elucidated [unpublished data]. Nevertheless, the previous study shows evidence of the possibility not only to assess glucose activity levels in the skin by means of calculating the $1030 \mathrm{~cm}^{-1}$ peak intensity values in measured spectra in vivo, but also to determine its changes in a dosedependent manner [11].

Among 11 subjects another two prominent glucosespecific peaks at $1041 \mathrm{~cm}^{-1}$ and $1118 \mathrm{~cm}^{-1}$ were identified and showed differences in their intensities in the measured spectra of non-diabetes, prediabetes and diabetes subjects. Based on that, two spectral patterns were seen for nondiabetes and for (pre-)diabetes subjects: $I_{1118}<I_{1041}$ for healthy, and $I_{1118}>I_{1041}$ for prediabetes and diabetes subjects.

In the present study ATR-FTIR spectroscopy has also demonstrated its potential to in vivo simultaneously monitor glucose-specific peaks at $1030 \mathrm{~cm}^{-1}, 1041 \mathrm{~cm}^{-1}, 1080 \mathrm{~cm}^{-1}$, $1118 \mathrm{~cm}^{-1}$ and $1153 \mathrm{~cm}^{-1}$ at different time intervals and after intake of different doses of glucose. Our shown results suggest that by means of measuring intensities of these peaks in the skin tissue we could not only determine differences in glucose activity levels in the skin of each subject, but could also to characterize and differentiate the activity of each glucose molecule between healthy, prediabetes and diabetes subjects in real time.

\section{CONCLUSION}

The results of the presented studies clearly demonstrate how vibrational spectroscopy techniques can be applied for identification, characterization and monitoring of several skin biomolecules in certain skin diseases and conditions, thus showing new possibilities to assess activity and severity of inflammation, activity of processes of carcinogenesis towards progression to malignancy, and activity levels of glucose metabolism in the skin tissue of healthy, prediabetes and diabetes subjects.

\section{FUTURE ASPECTS}

Although vibrational spectroscopy techniques has already started to be recognized with regard to their potentials to molecularly characterize and monitor numerous diseases and conditions, new fields of applications are still to be found. Among those are how to implement these techniques for direct real-time therapeutic interventions, based on already received information on molecular aspects of the diseases; how to monitor the effects of therapies based on molecular monitoring of severity and activity of pathological conditions and processes; and how to use normal and diseased vibrational spectra information for individualization of drug or treatment. Only the focused research and development of applications to further identify, characterize and monitor skin diseases and conditions undescribed at the molecular level will provide future ability to treat and control not only cancers, eczema and carbohydrate metabolism disorders, but also many others that await their medical needs and scientific opportunities.

\section{ACKNOWLEDGEMENTS}

Author NSE is thankful to Prof. P. Nielsen from Panum Institute, University of Copenhagen, Denmark, for his official invitation to present this paper at the $1^{\text {st }}$ International Conference on Drug Design and Discovery, Dubai, UAE, February 4-7, 2007. Author NSE is grateful to JSPS (Japan Society for Promotion and Science) for present financial support as a postdoctoral fellow; to Waseda University, Tokyo, Japan for sponsoring a presentation at the $1^{\text {st }}$ International Conference on Drug Design and Discovery, Dubai, UAE, February 4-7, 2007; to Monbusho (Japanese Ministry of Education) and to Danish Ministry of Education for financial support while at the Department of Dermatology, Bispebjerg Hospital, University of Copenhagen, Denmark, and at the Department of Dermatology, Tokushima University School of Medicine, Japan. She wants to thank again all those who helped during the studies on vibrational spectroscopy techniques: Dr. Gniadecka, Dr. Wessel, Prof. Wulf and the entire staff at the Department of Dermatology, Bispebjerg Hospital, University of Copenhagen, Denmark; Prof. N.I. Afanasyeva, Spectro Optical Sensing, Inc., USA; Fujimoto-san, Takeishi-san, all dermatologists in training for their support with skin tissue samples at the Department of Dermatology, Dr. Umehara at the Department of Orthopaedic Surgery, Prof. Yoshizaki and Dr. Ikehara at the Department of Physiology, Tokushima University School of Medicine; Dr. Huehne and staff at Kwansei-Gakuin University School of Science and Technology; Department of Physiology, Tokyo Medical University, Japan; Prof. Sota, students and members at the Department of Electrical Engineering and Bioscience, Waseda University, Japan.

\section{REFERENCES}

[1] Dukor, R.K. In Handbook of Vibrational Spectroscopy; Chalmers, J.M., Griffiths, P.R., Eds.; John Wiley and Sons Ltd.: 2002; Vol. 5, 3335-3361.

[2] Diem, M.; Boydston-White, S.; Chiriboga, L. Appl. Spectro., 1999, 53(4), 148A-161A

[3] Eikje Skrebova, N.; Aizawa, K.; Ozaki, Y.; Arase, S. Biotechnol. Ann. Rev., 2005; 11, 191-225.

[4] McIntosh, L.M.; Summers, R.; Jackson, M.; Mantsch, H.; Mansfield, J.R.; Howlett, M.; Crowson, A.N.; Toole, J.W.P. J. Invest. Dermatol., 2001, 116, 175-181.

[5] Schrader, B.; Dippel, B.; Fendel, S.; Keller, S.; Löchte, T.; Riedl, M.; Schulte, R.; Tatsch, E. J. Mol. Struct., 1997, 408/409, 23-31.

[6] Choo-Smith, L.-P.; Edwards, H.G.M.; Endtz, H.P.; Kros, J.M.; Heule, F.; Barr, H.; Robinson, J.S.; Bruining, H.A.; Puppels, G.J. Biopolymers (Biospectroscopy), 2002, 67, 1-9

[7] Gniadecka, M. Skin Res. Technol., 1997, 3, 139-146.

[8] Eikje Skrebova, N.; Ozaki, Y.; Aizawa, K.; Arase, S. J. Biomed. Optics, 2005; 10(1), 014013. 
[9] Eikje Skrebova, N. In Optical Technologies in Biophysics and Medicine II, Proceedings of SPIE Saratov Fall Meeting, Saratov, Russia, September 27-30, 2005; Tuchin, V.V., Ed.; International Society of Optical Engineering: Washington, USA, 2006, 6163, 616309 (invited paper).

[10] Eikje Skrebova, N. In Optical Technologies in Biophysics and Medicine II, Proceedings of SPIE Saratov Fall Meeting, Saratov, Russia, September 25-28, 2007; Tuchin, V.V., Ed.; International Society of Optical Engineering: Washington, USA, 2008, 6791-5, in press (invited paper).

[11] Eikje Skrebova, N.; Sota, T.; Aizawa, K. In Diagnostic Optical Spectroscopy in Biomedicine IV, Proceedings of SPIE European Conference on Biomedical Optics, Munich, Germany, 17-21 June, 2007; Schweitzer, D.; Fitzmaurice, M.; Eds; International Society of Optical Engineering: Washington, USA, 2007, 6628, 66281M.

[12] Wahlberg, J.E. In Textbook of Contact Dermatitis; Rycroft, R.J.G.; Mennè, T.; Frosch, P.J.; Benezra, C.; Eds.; Springer-Verlag: Berlin, Heidelberg, New York, 1995; Ch. 10.1, 239-268.

[13] Skrebova, N.; Ozaki, Y.; Arase, S. Subsurface Sens. Technol. Appl., 2002, 3, 19-34.

[14] Skrebova, N. In Subsurface Sensing Technologies \& Applications II, Proceedings of SPIE's $45^{\text {th }}$ Annual Meeting of the International
Society for Optical Engineering, San Diego, USA, July 31-August 3, 2000; Nguyen, C.; Ed.; International Society of Optical Engineering: Washington, USA, 2000; 4129, 218-230.

[15] Skrebova, N.; Aizawa, K.; Ozaki, Y.; Arase, S. In Smart Nondestructive Evaluation and Health Monitoring of Structural and Biological Systems, Proceedings of SPIE`s $8^{\text {th }}$ Annual International Symposium on Non-destructive Evaluation for Health Monitoring and Diagnostics, San Diego, California, USA, March 2-6, 2003; Kundu, T.; Ed.; International Society of Optical Engineering: Washington, USA, 2003; 5047; 378-385.

[16] Arndt, K.A. Manual of Dermatological Therapeutics (with essentials of diagnosis), $5^{\text {th }}$ ed.; Little, Brown and Company: Boston/ New York/ Toronto/ London, 1995.

[17] Lepoittevin, J.-P.; Basketter, D.A.; Goossens, A.; Karlberg, A.-T. Allergic Contact Dermatitis, Springer-Verlag: Berlin/Heidelberg/ New York, 1998.

[18] Jacobsen, A.D.T. Thesis, University of Odense, 1997

[19] Goldsmith, L.A. In Physiology, Biochemistry and Molecular Biology of the Skin, Oxford University Press: New York, 1991.

[20] Brancaleon, M.P.; Bamberg, M.P.; Sakamaki, T; Kollias, N. J. Invest. Dermatol., 2001, 116, 380-386.

(C) Eikje et al.; Licensee Bentham Open.

This is an open access article distributed under the terms of the Creative Commons Attribution License (http://creativecommons.org/license/by/2.5/), which permits unrestrictive use, distribution, and reproduction in any medium, provided the original work is properly cited. 